\title{
Renormalization Group evolution from on-shell SMEFT
}

\author{
Minyuan Jiang, ${ }^{a}$ Teng $\mathbf{M a}^{b}$ and Jing Shu ${ }^{a, c, d, e, f}$ \\ ${ }^{a}$ CAS Key Laboratory of Theoretical Physics, Institute of Theoretical Physics, \\ Chinese Academy of Sciences, \\ Beijing 100190, China \\ ${ }^{b}$ Physics Department, Technion - Israel Institute of Technology, \\ Haifa 3200003, Israel \\ ${ }^{c}$ School of Physical Sciences, University of Chinese Academy of Sciences, \\ Beijing 100049, China \\ ${ }^{d}$ CAS Center for Excellence in Particle Physics, Institute of Theoretical Physics, \\ Chinese Academy of Sciences, \\ Beijing 100049, China \\ ${ }^{e}$ Center for High Energy Physics, Physics Department, Peking University, \\ Beijing 100871, China \\ ${ }^{f}$ School of Fundamental Physics and Mathematical Sciences, \\ Hangzhou Institute for Advanced Study, University of Chinese Academy of Sciences, \\ Hangzhou 310024, China \\ E-mail: minyuan@itp.ac.cn, t.ma@campus.technion.ac.il, jshu@itp.ac.cn
}

ABstract: We describe the on-shell method to derive the Renormalization Group (RG) evolution of Wilson coefficients of high dimensional operators at one loop, which is a necessary part in the on-shell construction of the Standard Model Effective Field Theory (SMEFT), and exceptionally efficient based on the amplitude basis in hand. The UV divergence is obtained by firstly calculating the coefficients of scalar bubble integrals by unitary cuts, then subtracting the IR divergence in the massless bubbles, which can be easily read from the collinear factors we obtained for the Standard Model fields. Examples of deriving the anomalous dimensions at dimension six are presented in a pedagogical manner. We also give the results of contributions from the dimension- $8 H^{4} D^{4}$ operators to the running of $V^{+} V^{-} H^{2}$ operators, as well as the running of $B^{+} B^{-} H^{2} D^{2 n}$ from $H^{4} D^{2 n+4}$ for general $n$.

Keywords: Effective Field Theories, Renormalization Group, Scattering Amplitudes

ARXIV EPRINT: 2005.10261 


\section{Contents}

1 Introduction 1

2 The on-shell loop method based on unitary cut 3

2.1 Unitarity cut and bubble coefficients 3

2.2 Extraction of bubble coefficient 4

3 Collinear divergence $\quad 6$

3.1 Example to deriving the collinear factor for Higgs and Hypercharge gauge boson 6

3.2 Complete collinear factors of SM fields 8

4 Examples for calculation of anomalous dimension matrix $\quad 8$

$4.1 \mathcal{O}_{H B} \quad 8$

$4.2 F^{3}$ type operators 11

5 Anomalous dimensions at dimension 8: the $V^{+} V^{-} H^{\dagger} H$ example 12

6 Universal results for anomalous dimension 13

$\begin{array}{lll}7 \text { Conclusion } & 14\end{array}$

$\begin{array}{ll}\text { A The anomalous dimension of } O_{e W} & 16\end{array}$

\section{Introduction}

The discovery of the Higgs with a mass around $125 \mathrm{GeV}[1,2]$ completes the last missing piece of the Standard Model (SM) and indicates that the SM precisely describes the fundamental interactions at lower energy scale. But with the discovery of Higgs, the Higgs naturalness problem is still mysterious and remains to be solved in the next decades. To solve this problem, the new physics (NP) and symmetry should be introduced at TeV scale, such as SUSY [3, 4] and composite Higgs models [5-7]. But so far the $14 \mathrm{TeV}$ LHC still did not find any new physics, which may indicate that the NP scale is so high that beyond the reach of current experiment searches. With such a high NP scale, the precise measurements of the SM interactions at lower scale is an available way to search for the hints of NP, which can be well parametrized by high dimensional operators of the SM Effective Filed Theory (SMEFT). So it becomes important to understand the SMEFT for the search of NP imprints. Since the running of Wilson coefficients can significantly affect the contributions of NP to SM fields interactions at loop level, the anomalous dimensions 
of effective operators are crucial for correctly calculating the SMEFT processes without loosing any infrared informations of the NP.

Recently it was found that on-shell scattering amplitudes have remarkable advantages for the study of SMEFT, comparing with the traditional Lagrangian language. The high dimension operators can be described by unfactorizable amplitudes [8,9], called amplitude basis, without boring with the redundancies from the equation of motion and integration by part (these redundancies are automatically removed by the intrinsic properties of onshell method: on-shell condition and momentum conservation). With this new bases, the calculation in SMEFT can be implemented without referring to the Lagrangian. Some surprising relations and properties of EFTs, which are not manifest in quantum field theory, can be easily seen via this method. For example, some EFTs can be described by scattering equations [10] uniformly or constructed/ classified systematically from soft limits [11-13]. The running of Wilson coefficients of SMEFT can be strongly constrained by selection rules [14-16] based on unitarity cut method. Since the on-shell scattering amplitudes are only described by the physical freedoms of external legs, the calculations of SMEFT can be much more efficient via on-shell method without involving gauge fixing and ghosts. Moreover, the one loop amplitudes can be decomposed into the sum of a basis of scalar integrals plus rational functions, and the coefficients of the scalar integrals are determined by the product of the tree-level on-shell amplitudes from the generalized unitary cuts [1719]. So the one loop amplitudes can be obtained through simple tree-level calculations without involving any loop calculations. Since only the bubble integrals are UV divergent, the anomalous dimension matrix can be determined by the massive and massless bubble integral coefficients, which can be easily obtained by Stokes's Theorem [20] or other methods [11, 21-23] (massive bubble integrals) and collinear divergences of tree level amplitudes (massless bubble integrals) [24-26]. Notice that UV divergences from massless bubble integrals are universal and only determined by renormalizable interactions so they can be directly read out without any calculations [26]. Comparing with the existing calculation of the anomalous dimension matrix of dimension six [27-31] via Feynman diagram, on-shell method is very convenient and powerful for SMEFT study, especially when applied in the calculations involving higher dimension operators.

In this paper we demonstrate how to use the on-shell method to derive the anomalous dimension matrix via tree-level amplitudes and give some non-trivial examples, such as $F^{3}$ type operators and dimension 8 operators (the complete dimension 8 operator basis can be found in $[32,33])$. Since the UV divergence from a massless bubble integral is universal (only depends on the external legs attached to this bubble diagram), we list all the UV divergent factors from massless bubbles for all the SM fields. So people can directly use these results to calculate the renormalization of SMEFT operators at one loop level without calculating this kind of UV divergences again. We find that the custodial symmetry can also explain some zeros in anomalous dimension matrices, which can not be explained by the existing selection rules. Based on unitary cuts, the anomalous dimension matrices of the operators with arbitrary dimensions that contribute to $2 \rightarrow 2$ processes can be easily expressed in universal forms and we explicitly show the universal expressions for the running of $B^{+} B^{-} H^{2} D^{n}$ type operators generated from the insertion of general $H^{4} D^{2 n+4}$ type operators at one-loop level. 
The structure of this paper is organized as follows. A detailed discussion about the anomalous dimension calculation in SMEFT via the on-shell method is presented in section 2 , and some examples are shown in section 4 . The anomalous dimension matrix for dimension 8 amplitude basis $V^{+} V^{-} H H^{\dagger}$ is obtained in section 5. We show the universal results of anomalous dimensions for general amplitude basis $B^{+} B^{-} H^{2} D^{2 n}$ in section 6 and conclude in section 7. A simple example of deriving collinear divergent factor and detailed calculation of the anomalous dimension of $\mathcal{O}_{e W}$ via on-shell method are presented in appendix 3.1 and appendix A.

\section{The on-shell loop method based on unitary cut}

Since the renormalization of on-shell SMEFT is induced by UV divergent part of the amplitudes, in this section we explain how to use unitarity cut to derive the UV divergences in one-loop amplitudes.

The non-renormalizable interactions of the on-shell SMEFT can be described by the amplitude basis $\sum_{i} c_{i} \mathcal{M}_{\mathcal{O}^{i}}$, where $c_{i}$ is the Wilson coefficient. To obtain the RG equations for $c_{i}$, we consider the amplitude which receives tree-level contribution from $\mathcal{M}_{\mathcal{O}^{i}}$ as well as loop contributions with another amplitude basis $\mathcal{M}_{\mathcal{O}^{j}}$ insertion. The full amplitude takes the form of

$$
\mathcal{A}_{i} \sim c_{i}(\mu)-\gamma_{i j} \frac{1}{16 \pi^{2}} c_{j}(\mu)\left(\frac{1}{2 \epsilon}+\log \mu+\ldots\right),
$$

where the terms $\frac{1}{2 \epsilon}+\log \mu$ come from the UV divergence and $\mu$ is the renormalization scale. By demanding the full amplitude being independent of the scale $\mu$, one directly obtains renormalization group (RG) equation

$$
\frac{\mathrm{d} c_{i}(\mu)}{\mathrm{d} \log \mu}=\sum_{j} \frac{1}{16 \pi^{2}} \gamma_{i j} c_{j}
$$

where $\gamma_{i j}$ is the anomalous dimension matrix governing the RG running.

\subsection{Unitarity cut and bubble coefficients}

To extract the UV divergence in the one-loop amplitude, a convenient way is to decompose it into the combination of a basis of scalar integrals including boxes, triangles and bubbles plus rational functions $[17,18]$

$$
\mathcal{A}^{1 \text {-loop }}=\sum_{k} C_{4}^{k} I_{4}^{k}+\sum_{j} C_{3}^{j} I_{3}^{j}+\sum_{k} C_{2}^{i} I_{2}^{i}+R
$$

Here the index $i$ ( $j$ or $k$ ) labels the distinct integrals with different partition of the external legs. These integrals capture the branch cuts of the loop amplitudes and their coefficients $C_{4,3,2}^{i}$ can be obtained from tree level amplitudes by generalized unitary cut [34-36] . The scalar bubbles are the only UV divergent integrals in four dimensions. With dimension regularization it takes the form:

$$
I_{2}^{i} \equiv-i \int \frac{d^{d} l}{(2 \pi)^{d}} \frac{1}{l^{2}(l-K)^{2}}=\frac{1}{(4 \pi)^{2}}\left(\frac{1}{\epsilon}-\log \frac{-K^{2}}{\mu^{2}}+\ldots\right) .
$$




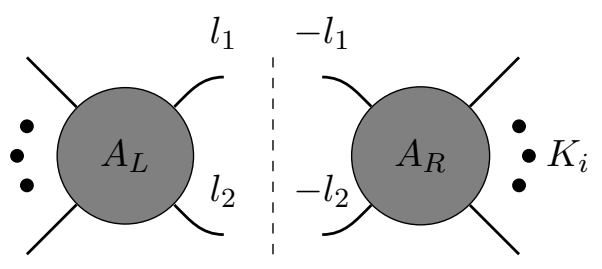

Figure 1. $K_{i}$-channel double cut.

where $D=4-2 \epsilon$. So the only job to derive anomalous dimension matrix is to extract the bubble coefficients. For the massive bubbles $(K>0)$, the coefficients can be easily obtained by unitarity cut. In the following section, we will briefly discuss about this method.

\subsection{Extraction of bubble coefficient}

The basic idea of the extraction of bubble coefficient is that the contributions from bubbles to the double cuts of the loop amplitude are just simple rational functions while the cuts on the higher point basis $I_{m}$ with $m>2$ give logarithmic functions because the un-cut propagators in basis $I_{m}$ will make the variables of integration appearing in the denominators. According to the one-loop amplitude decomposition in eq. (2.3), the bubble coefficient $C_{2}$ should be equal to the rational terms of the double cuts of amplitude $\mathcal{A}^{1 \text {-loop }}$ divided by double cuts of $I_{2}$. So the only job to obtain $C_{2}$ is to extract these rational terms from double cuts of $\mathcal{A}^{\text {1-loop}}$, which can be efficiently done through the indefinite integration by Hermite Polynomial Reduction [20]. In the following, we briefly introduce the method proposed in [20] to extract the coefficient of massive bubbles.

As said before, to extract the bubble coefficient $C_{2}^{i}$, the $K_{i}$-channel double-cut should be implemented to $\mathcal{A}^{1 \text {-loop }}$ in eq. (2.3) as illustrated in figure 1, the left hand side of this equation becomes

$$
\operatorname{Cut}_{K_{i}}\left[\mathcal{A}^{1 \text {-loop }}\right]=\int d \operatorname{LIPS}_{i} \sum_{h_{i}} \mathcal{A}_{L}^{\text {tree }}\left(l_{1}, l_{2}, h_{i}\right) \mathcal{A}_{R}^{\text {tree }}\left(-l_{1},-l_{2},-h_{i}\right)
$$

where $d$ LIPS $_{i}=d^{4} l_{1} d^{4} l_{2} \delta^{(+)}\left(l_{1}^{2}\right) \delta^{(+)}\left(l_{2}^{2}\right) \delta^{(4)}\left(l_{1}+l_{2}-K_{i}\right)$ is the Lorentz-invariant phase space associated with the $K_{i}$-channel cut, $K_{i}$ is the total momentum of the particles at one side of the cut, $l_{1,2}^{\mu}$ is momentum of the cut loop propagators, $\mathcal{A}_{L}^{\text {tree }}, \mathcal{A}_{R}^{\text {tree }}$ are tree level amplitudes on each side of the cut and $h_{i}$ is the polarization configuration of the cut internal legs. Since the loop propagators in the bubble integrations are both on-shell by imposing the doublecut, the double-cut of $I_{2}^{i}$ is just a constant (see eq. (2.12)). Thus the bubble coefficient should be proportional to the rational terms of double cut amplitude $\operatorname{Cut}_{K_{i}}\left[\mathcal{A}^{1 \text {-loop }}\right]$. With the one-loop amplitude decomposition, the bubble coefficients is given by

$$
C_{2}^{i}=\frac{1}{\Delta I_{2}^{i}} \text { Rational }\left[\int d \operatorname{LIPS}_{i} \sum_{h_{i}} \mathcal{A}_{L}^{\text {tree }} \mathcal{A}_{R}^{\text {tree }}\right],
$$

where the factor $\Delta I_{2}^{i}=-2 \pi i$ is the double-cut of bubble integral $I_{2}^{i}$ (will be seen in the following discussion). To efficiently calculate the phase space integral, since the loop momentum is on-shell enforced by unitary cut, the loop momentum can be parametrized in 
term of massless spinors [37],

$$
l_{1}^{\mu} \sigma_{\mu, a \dot{a}} \equiv\left(l_{1}\right)_{a \dot{a}}=t|\lambda\rangle_{a}\left[\left.\lambda\right|_{\dot{a}},\right.
$$

where $\sigma_{\mu}=\left\{1, \sigma_{i}\right\}, \sigma_{i}$ is Paul matrix, $|\lambda\rangle_{a}\left(\left[\left.\lambda\right|_{\dot{a}}\right)\right.$ is the massless two-component spinor of Lorentz subgroup $\mathrm{SU}(2)_{L}\left(\mathrm{SU}(2)_{R}\right)$ (more details about spinors can be found in [38]). With this parametrization, the phase space integral can be written in terms of spinor product [37],

$$
\int d \operatorname{LIPS}_{i}=\int t d t \int_{\left(|\lambda\rangle_{a}\right)^{*}=\left[\left.\lambda\right|_{\dot{a}}\right.} \frac{\langle\lambda d \lambda\rangle[\lambda d \lambda]}{\left\langle l\left|K_{i}\right| l\right]} \delta\left(t-\frac{K_{i}^{2}}{\left\langle l\left|K_{i}\right| l\right]}\right),
$$

where $\left.\left.\left\langle\lambda_{j} \lambda_{i}\right\rangle=\epsilon^{a b}\left|\lambda_{i}\right\rangle_{a}\left|\lambda_{j}\right\rangle_{b}\left(\left[\lambda_{j} \lambda_{i}\right]=\epsilon^{a b} \mid \lambda_{i}\right]_{a} \mid \lambda_{j}\right]_{b}\right)$ is spinor product, which is Lorentz scalar [38]. Notice that since the loop momentum is real, the integration contour should satisfy the condition $\left(|\lambda\rangle_{a}\right)^{*}=\left[\left.\lambda\right|_{\dot{a}}\right.$. Then two-component spinor variable $\left.|\lambda\rangle(\mid \lambda]\right)$ can be decomposed with a basis of two massless spinors,

$$
|\lambda\rangle=|p\rangle+z|q\rangle, \mid \lambda]=\mid p]+\bar{z} \mid q]
$$

where $|p, q\rangle(\mid p, q])$ is the spinor for null momentum $p_{\mu}$ and $q_{\mu}$, which are required to satisfy $K_{\mu}^{i}=p_{\mu}+q_{\mu}$. Notice that the choice of null momentum basis $p$ and $q$ is arbitrary and can not be fixed by the condition $K_{\mu}^{i}=p_{\mu}+q_{\mu}$, while the final result should be independent of a particular choice. With this parametrization, the phase space integral can be expressed in term of the integration on the complex plane of $z$,

$$
\int d \operatorname{LIPS}_{i}=\iint_{D} d z \wedge d \bar{z} \int d t t^{2} \delta\left(t-\frac{1}{(1+z \bar{z})}\right) .
$$

Here the integration domain $D$ is the whole complex plane. It is proved in [20] with Stokes' Theorem that this integration can be effectively performed as,

$$
\int d \operatorname{LIPS}_{i}=\oint d z \int d \bar{z} \int d t t^{2} \delta\left(t-\frac{1}{(1+z \bar{z})}\right) .
$$

Here the integration over $\bar{z}$ is indefinite and the primitive function of the integrand with respect to $\bar{z}$ should not contain any functions purely of $z$ which have non-vanishing residues. Notice when doing $\bar{z}$ integration, $z$ should be treated as independent variable. We then do contour integration over $z$ at the infinity. When doing the $z$-integral via Cauchy's Residue theorem, $\bar{z}$ appearing in the residues should take the corresponding complex-conjugate values of $z$ at the poles. The rigorous definition of the $z$ and $\bar{z}$ integral and more details about the prove can be found in [20]. Then we can explicitly calculate double-cut of the bubble integral through above parametrization and find that it is just a constant,

$$
\Delta I_{2}^{i} \equiv \operatorname{Cut}\left[I_{2}^{i}\right]=\oint d z \int d \bar{z} \int t^{2} d t \delta\left(t-\frac{1}{(1+z \bar{z})}\right)=\int d z \frac{-1}{(1+z \bar{z}) z}=-2 \pi i .
$$

In the last two step, we first integrate over $\bar{z}$ by keep $z$ as independent variable and then sum over the residues at all poles of $z$ with substituting the corresponding complex conjugate value of $z$ into $\bar{z}$ appearing in the residues. Under this parametrization the cut amplitude becomes

$$
\Delta_{i} \equiv \int d \operatorname{LIPS}_{i} \sum_{h_{i}} \mathcal{A}_{L}^{\text {tree }} \mathcal{A}_{R}^{\text {tree }}=\left.\oint d z \int d \bar{z} t^{2} \sum_{h_{i}} \mathcal{A}_{L}(t, z, \bar{z}) \mathcal{A}_{R}(t, z, \bar{z})\right|_{t=\frac{1}{1+z \bar{z}}} .
$$


After performing the $z$-integration via Cauchy's Residue Theorem, finally $C_{2}^{i}$ in eq. (2.6) can be easily evaluated via the following expression,

$$
C_{2}=\frac{\Delta_{i}^{\text {Rational }}}{-2 \pi i}=-\operatorname{Res}_{z=0} F^{\text {Rational }}(z, \bar{z})-\operatorname{Res}_{z \neq 0} F^{\text {Rational }}(z, \bar{z}),
$$

where $\Delta_{i}^{\text {Rational }}$ denotes the rational terms of the cut amplitude $\Delta_{i}, F^{\text {Rational }}$ is the rational part of primitive function $F(z, \bar{z})=\left.\int d \bar{z} t^{2} \sum_{h_{i}} \mathcal{A}_{L}(t, z, \bar{z}) \mathcal{A}_{R}(t, z, \bar{z})\right|_{t=1 /(1+z \bar{z})}$.

\section{Collinear divergence}

Using unitary cut we can not get the full UV divergences of loop amplitudes. Only the coefficients of massive bubble $\left(K_{i}^{2}>0\right)$ can be obtained via unitary cut. Massless bubbles with $K_{i}^{2}=0$ also contain UV divergences, which simply vanish in dimension regularization due to the cancellation between UV and collinear IR divergence [11, 14, 21]. Since the physical cross section is free of collinear divergences, the collinear divergences of the tree amplitudes must be cancelled by collinear loop IR divergences. So the UV divergence in massless bubbles can be extracted by calculating collinear divergences of the corresponding tree amplitudes. The one loop collinear IR divergence can be parametrized as [24-26]

$$
\mathcal{A}_{\mathrm{n}, \mathrm{col}}^{1 \text {-loo }}=-\left(\frac{1}{4 \pi}\right)^{2} \sum_{a}^{n} \frac{\gamma(a)}{\epsilon} \mathcal{A}^{\text {tree }},
$$

where the sum is over all external legs, $\epsilon$ is regularization parameter, and $\gamma(a)$ is the collinear factor associated with particle $a$. We want to emphasize that the collinear factors only depend on the external legs and are universal for SMEFT. In the following discussions, we will take scalar $\mathrm{U}(1)_{Y}$ gauge theory as an example to explicitly show how to derive the collinear factors generally.

\subsection{Example to deriving the collinear factor for Higgs and Hypercharge gauge boson}

In this subsection, we show how to get the collinear divergent factor for Higgs in $\mathrm{U}(1)_{Y}$ gauge theory, following the procedure in $[24,25]$. To get the collinear factor of Higgs leg generated from $\mathrm{U}(1)_{Y}$ gauge interactions, we should look at the scattering amplitudes containing the 3-vertex of Higgs gauge interactions, such as the four point amplitude of $H H^{\dagger} \rightarrow B^{+} B^{-}$(the superscript \pm of $B$ represents helicity). To get the collinear factor associated with Higgs field, the external legs attaching with internal Higgs propagator should be collinear such that Higgs is on shell. In the process $H H^{\dagger} \rightarrow B^{+} B^{-}$, we suppose the external Higgs leg $H$ with momentum $p_{1}$ and $B^{+}$with momentum $p_{3}$ attach to the Higgs propagator, so if $p_{1}$ and $p_{3}$ become collinear, the Higgs propagator become on-shell and the amplitude are divergent. We can parametrize the their momentum as

$$
|1\rangle=\sqrt{z}|P\rangle \quad|3\rangle=\sqrt{1-z}|P\rangle
$$

where $0<z<1$ and $P$ is the momentum of Higgs propagator. The four point amplitudes $\mathcal{A}\left(H H^{\dagger} B^{+} B^{-}\right)$becomes divergent and can factorize into the product of three point 
amplitude and a singular splitting function

$$
\mathcal{A}\left(H\left(p_{1}\right) H^{\dagger}\left(p_{2}\right) B^{+}\left(p_{3}\right) B^{-}\left(p_{4}\right)\right) \rightarrow \operatorname{Split}_{H}\left(H, B^{+}\right) \mathcal{A}\left(H(P) H^{\dagger}\left(p_{2}\right) B^{-}\left(p_{4}\right)\right)
$$

where the spliting function $\operatorname{Split}_{H^{\dagger}}\left(H\left(p_{1}\right), B^{+}\left(p_{3}\right)\right)=\sqrt{2} g_{1} y_{h} \frac{\sqrt{z}}{\sqrt{1-z}\langle 13\rangle}, y_{h}$ is the Hypercharge of Higgs doublet and the three point amplitude

$$
\mathcal{A}\left(H(P) H^{\dagger}\left(p_{2}\right) B^{-}\left(p_{4}\right)\right)=\sqrt{2} g_{1} y_{h} \frac{\langle 4 P\rangle\langle 42\rangle}{\langle P 2\rangle} .
$$

We can find that the collinear factor $c_{F}^{H B \rightarrow H^{\dagger}}$ which describes collinear singularity of the amplitude square can be expressed as

$$
c_{F}^{H B \rightarrow H^{\dagger}}=\sum_{i= \pm} \operatorname{Split}_{H^{\dagger}}\left(H\left(p_{1}\right), B^{i}\left(p_{3}\right)\right) \operatorname{Split}_{H^{\dagger}}^{\dagger}\left(H\left(p_{1}\right), B^{i}\left(p_{3}\right)\right)=\frac{4 g_{1}^{2} y_{h}^{2}}{\langle 13\rangle[13]} \frac{z}{1-z} .
$$

Notice that the contributions from different polarization of gauge boson $B_{\mu}$ should be included. The phase space of these two collinear legs, which can regularize the collinear factor $c_{F}^{H B \rightarrow H^{\dagger}}$ in amplitude square, can be expressed as [24]

$$
d P_{\mathrm{col}}^{\epsilon}\left(p_{1}, p_{3}, z\right)=\frac{(4 \pi)^{\epsilon}}{16 \pi^{2} \Gamma(1-\epsilon)} d s_{13} d z\left[s_{13} z(1-z)\right]^{-\epsilon} \theta\left(s_{\min }-s_{13}\right) .
$$

Integrating the collinear factor over this phase space, we can get the collinear divergent factor of the tree-level cross section of the process $H H^{\dagger} \rightarrow B^{+} B^{-}$,

$$
\int c_{F}^{H B \rightarrow H^{\dagger}} d P_{\mathrm{col}}^{\epsilon}\left(p_{1}, p_{3}, z\right)=\frac{g_{1}^{2} y_{h}^{2}}{4 \pi^{2}} \frac{1}{\Gamma(1-\epsilon)} \frac{1}{\epsilon} .
$$

The tree level collinear divergences should be cancelled by collinear IR divergences of oneloop level amplitudes. If the one-loop IR divergences are parametrized as in eq. (3.1), the one-loop collinear IR divergent factor of Higgs leg is $-\gamma(H) /\left(16 \pi^{2} \epsilon\right)$. By comparing with these two divergent factors, we can get the collinear factor from Higgs leg (only include $\mathrm{U}(1)_{Y}$ gauge corrections), ${ }^{1}$

$$
\gamma(H)=2 g_{1}^{2} y_{h}^{2}
$$

Following the same procedure, we can extract the splitting function for vertex $H\left(p_{1}\right) H^{\dagger}\left(p_{2}\right) \rightarrow B$ from the process $H^{a} H^{\dagger \dot{b}} \rightarrow H^{c} H^{\dagger \dot{d}}, \operatorname{Split}_{B^{ \pm}}\left(H^{a}, H^{\dagger \dot{b}}\right)=$ $\delta^{a \dot{b}} \sqrt{2} g_{1} y_{h} \sqrt{z(1-z)} /\langle 12\rangle$. The collinear factor can be given by

$$
c_{F}^{H\left(p_{1}\right) H^{\dagger}\left(p_{2}\right) \rightarrow B^{ \pm}}=\sum_{a, \dot{b}} \operatorname{Split}_{B^{ \pm}}\left(H^{a}, H^{\dagger \dot{b}}\right) \operatorname{Split}_{B^{ \pm}}^{\dagger}\left(H^{a}, H^{\dagger \dot{b}}\right)=\frac{4 g_{1}^{2} y_{h}^{2}}{\langle 12\rangle[12]} z(1-z) .
$$

After doing the same integration as in eq. (3.7), we can easily get the collinear factor from $\mathrm{U}(1)_{Y}$ gauge boson legs (only include Higgs doublet corrections)

$$
\gamma\left(B^{ \pm}\right)=-\frac{g_{1}^{2} y_{h}^{2}}{3}
$$

\footnotetext{
${ }^{1}$ For the cross section of a process, the collinear divergences from the tree level amplitude square are cancelled by the collinear IR divergences from the product of the tree and one-loop level scattering amplitude so the collinear divergent factor of tree level cross section is twice times bigger than the one loop collinear IR divergent factor.
} 


\subsection{Complete collinear factors of SM fields}

Following the same procedure, we can easily get all the collinear factors associated with all the SM fields by simple tree level calculations. Since these factors are universal, we list all of them in eq. (3.11). Thus, for any SMEFT one-loop calculation, the UV divergences of massless bubbles can be directly read from the list below without any calculation.

$$
\begin{aligned}
\gamma\left(H^{a}\right) & =\gamma\left(H^{\dagger \dot{a}}\right)=2 y_{h}^{2} g_{1}^{2}-\frac{1}{2} \operatorname{Tr}\left[N_{c} Y_{u}^{\dagger} Y_{u}+N_{c} Y_{d}^{\dagger} Y_{d}+Y_{e}^{\dagger} Y_{e}\right]+2 g_{2}^{2} C_{2}(2), \\
\gamma\left(B^{ \pm}\right) & =-\frac{g_{1}^{2}}{3}\left[n_{g} N_{c}\left(y_{q}^{2}+y_{u}^{2}+y_{d}^{2}\right)+n_{g}\left(y_{\ell}^{2}+y_{e}^{2}\right)+y_{h}^{2}\right], \\
\gamma\left(W^{a \pm}\right) & =g_{2}^{2}\left[\frac{11}{3}-\frac{1}{3}\left(\frac{n_{g}}{2} N_{c}+\frac{n_{g}}{2}+\frac{1}{4}\right)\right], \\
\gamma\left(g^{ \pm}\right) & =g_{3}^{2}\left(\frac{11 N_{c}}{6}-\frac{1}{3} n_{g}\right), \\
\gamma(\ell) & =\frac{3}{2} g_{2}^{2} C_{2}(2)+\frac{3}{2} y_{\ell}^{2} g_{1}^{2}-\frac{1}{4} Y_{e}^{\dagger} Y_{e}, \\
\gamma(e) & =\frac{3}{2} y_{e}^{2} g_{1}^{2}-\frac{1}{2} Y_{e}^{\dagger} Y_{e} \\
\gamma(q) & =\frac{3}{2} g_{3}^{2} C_{2}\left(N_{c}\right)+\frac{3}{2} g_{2}^{2} C_{2}(2)+\frac{3}{2} y_{q}^{2} g_{1}^{2}-\frac{1}{4}\left(Y_{u}^{\dagger} Y_{u}+Y_{d}^{\dagger} Y_{d}\right), \\
\gamma(u) & =\frac{3}{2} g_{3} C_{2}\left(N_{c}\right)+\frac{3}{2} y_{u}^{2} g_{1}^{2}-\frac{1}{2} Y_{u}^{\dagger} Y_{u}, \\
\gamma(d) & =\frac{3}{2} g_{3} C_{2}\left(N_{c}\right)+\frac{3}{2} y_{u}^{2} g_{1}^{2}-\frac{1}{2} Y_{d}^{\dagger} Y_{d} .
\end{aligned}
$$

Here $q$ and $l$ are $\mathrm{SU}(2)$ doublets for left hand quarks and leptons, while $u, d$ and $e$ are right hand singlets. $g_{3}, g_{2}$ and $g_{1}$ are the gauge couplings of $\mathrm{SU}(3)_{C} \times \mathrm{SU}(2)_{L} \times \mathrm{U}(1)_{Y}$, with $y_{i}$ being the hypercharge. $Y_{u}, Y_{d}$ and $Y_{e}$ are Yukawa couplings. $N_{c}=3$ is QCD color number; $n_{g}=3$ is the number of generations. And $C_{2}(N)=\frac{N^{2}-1}{2 N}$.

Combining the UV divergences from massive bubbles with collinear IR divergences, we can finally obtain the anomalous dimension matrix correctly. In the next section we will give some non-trivial examples to clearly show how to get the anomalous dimension matrix systematically via on-shell method.

\section{Examples for calculation of anomalous dimension matrix}

In this section we give some examples to demonstrate the on-shell loop method for calculating the RG running of SMEFT in detail.

\section{$4.1 \mathcal{O}_{H B}$}

We first focus on a simplest case: the contributions proportional to $\mathrm{U}(1)_{Y}$ gauge interactions to the running of the dimension 6 operator $\mathcal{O}_{H B}=H^{\dagger} H B^{\mu \nu} B_{\mu \nu}$. In the amplitude basis, this operator corresponds to the local amplitude

$$
\mathcal{A}\left(B^{+}, B^{+}, H^{\alpha}, H^{\dagger \dot{\beta}}\right)=2 C_{H B} \delta^{\alpha \dot{\beta}}[12]^{2} .
$$



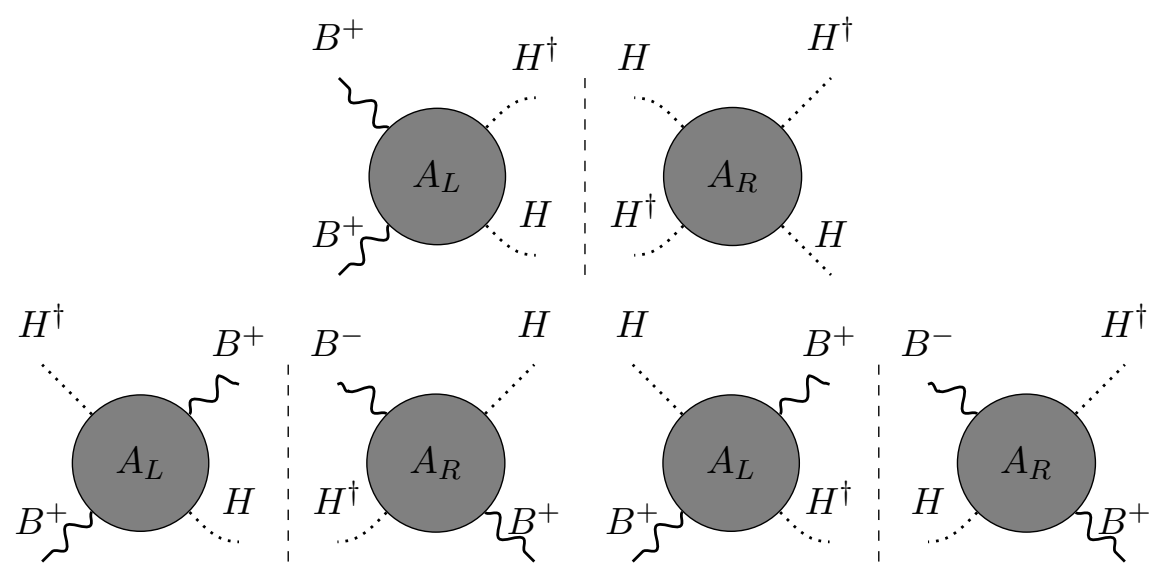

Figure 2. Unitary cuts in calculating the running of $O_{H B}$. The upper plot is the $s$ channel cut and the lower two plots are $t$ channel cuts.

The Higgs $\mathrm{U}(1)_{Y}$ gauge interactions and quartic term can be expressed as

$$
\mathcal{L}=-\frac{1}{4} B_{\mu \nu} B^{\mu \nu}+\left|D_{\mu} H\right|^{2}-\frac{1}{4} \lambda|H|^{4} .
$$

where $D_{\mu}=\partial_{\mu}-i g_{1} y_{h} B_{\mu}$.

Now let's consider the amplitude $\mathcal{A}\left(B^{+}, B^{+}, H^{\dagger \dot{\alpha}}, H^{\alpha}\right)$ at one loop. As shown in figure 2, applying different double cuts, this amplitude are separated into dimension 6 part and dimension 4 part. The dimension 6 part can be read from (4.1) and the dimension 4 part can be derived from (4.2),

$$
\begin{aligned}
\mathcal{A}^{4}\left(H^{\alpha} H^{\dagger \dot{\alpha}} B^{+} B^{-}\right)= & -2 y_{h}^{2} g_{1}^{2} \delta^{\alpha \dot{\alpha}} \frac{\langle 14\rangle\langle 24\rangle}{\langle 13\rangle\langle 23\rangle} \\
\mathcal{A}^{4}\left(H^{\alpha} H^{\dagger \dot{\alpha}} H^{\beta} H^{\dagger \dot{\beta}}\right)= & \left(\delta^{\alpha \dot{\alpha}} \delta^{\beta \dot{\beta}}+\delta^{\alpha \dot{\beta}} \delta^{\beta \dot{\alpha}}\right)\left(-\frac{\lambda}{2}+y_{h}^{2} g_{1}^{2}\right) \\
& +2 y_{h}^{2} g_{1}^{2}\left[\delta^{\alpha \dot{\alpha}} \delta^{\beta \dot{\beta}} \frac{\langle 24\rangle[24]}{\langle 12\rangle[12]}+\delta^{\alpha \dot{\beta}} \delta^{\beta \dot{\alpha}} \frac{\langle 24\rangle[24]}{\langle 14\rangle[14]}\right] .
\end{aligned}
$$

First consider s-channel cut (the upper plot in figure 2) and the product of the two cut amplitudes can be expressed as

$$
\begin{aligned}
& \operatorname{Cut}_{12}\left[\mathcal{A}\left(B^{+}\left(p_{1}\right) B^{+}\left(p_{2}\right) H^{\dagger \dot{\alpha}}\left(p_{3}\right) H^{\alpha}\right)\left(p_{4}\right)\right] \\
& =\int d \operatorname{LIPS} \mathcal{A}_{L}\left(B^{+}\left(p_{1}\right) B^{+}\left(p_{2}\right) H^{\beta}\left(l_{1}\right) H^{\dagger \dot{\beta}}\left(l_{2}\right)\right) \delta^{\beta \dot{\sigma}} \delta^{\sigma \dot{\beta}} \mathcal{A}_{R}\left(H^{\dagger \dot{\sigma}}\left(-l_{1}\right) H^{\sigma}\left(-l_{2}\right) H^{\dagger \dot{\alpha}}\left(p_{3}\right) H^{\alpha}\left(p_{4}\right)\right) \\
& =\int d \operatorname{LIPS} C_{H B} \delta^{\sigma \dot{\sigma}}[12]^{2}\left[\left(\delta^{\sigma \dot{\sigma}} \delta^{\alpha \dot{\alpha}}+\delta^{\sigma \dot{\alpha}} \delta^{\alpha \dot{\sigma}}\right)\left(-\frac{\lambda}{2}+y_{h}^{2} g_{1}^{2}\right)\right. \\
& \left.\quad+2 y_{h}^{2} g_{1}^{2}\left(\delta^{\sigma \dot{\sigma}} \delta^{\alpha \dot{\alpha}} \frac{\left\langle-l_{2} 3\right\rangle\left[-l_{2} 3\right]}{\left\langle-l_{1}-l_{2}\right\rangle\left[-l_{1}-l_{2}\right]}+\delta^{\sigma \dot{\alpha}} \delta^{\alpha \dot{\sigma}} \frac{\left\langle-l_{2} 3\right\rangle\left[-l_{2} 3\right]}{\left\langle-l_{1} 3\right\rangle\left[-l_{1} 3\right]}\right)\right],
\end{aligned}
$$

where $l_{1,2}$ is the momentum of the cut internal Higgs leg. We then use the relations $|-l\rangle=i|l\rangle, \mid-l]=i \mid l]^{2}$ and $l_{1}+l_{2}=p_{3}+p_{4}$ to express it as function of $l_{1}$. Following the

\footnotetext{
${ }^{2}$ For internal fermion leg, the complex factor $i$ can be removed because the fermion statistic cancels the minus sign from momentum flipping.
} 
procedure presented in the previous section, we should parametrize the loop momentum as

$$
\left.\left.\left.\left|l_{1}\right\rangle=\sqrt{t}(|3\rangle+z|4\rangle) \quad \mid l_{1}\right]=\sqrt{t}(\mid 3]+\bar{z} \mid 4\right]\right),
$$

and then the bubble coefficient can be extracted through Stokes' Theorem

$$
\begin{aligned}
\Delta_{12}= & {\left.[12]^{2} \delta^{\alpha \dot{\alpha}} \oint d z \int d \bar{z} t^{2}\left(\left(-\frac{3}{2} \lambda+3 y_{h}^{2} g_{1}^{2}\right)+2 y_{h}^{2} g_{1}^{2}\left(-2 t^{3}+\frac{1}{z \bar{z}} t^{2}\right)\right)\right|_{t=(1+z \bar{z})^{-1}} } \\
=[12]^{2} \delta^{\alpha \dot{\alpha}} \oint d z( & \left(-\frac{3}{2} \lambda+3 y_{h}^{2} g_{1}^{2}\right) \frac{-1}{z(1+z \bar{z})} \\
& \left.\quad+2 y_{h}^{2} g_{1}^{2}\left(\frac{1}{z(1+z \bar{z})^{2}}+\frac{1}{z(1+z \bar{z})}+\frac{\log (-z \bar{z})-\log (1+z \bar{z})}{z}\right)\right) \\
= & 2 \pi i[12]^{2} \delta^{\alpha \dot{\alpha}}\left(\frac{3}{2} \lambda+3 y_{h}^{2} g_{1}^{2}\right) .
\end{aligned}
$$

In the last step we discard the $\log$ term and take the residue at $z=0$ and set $\bar{z}=z$. Finally we can get the bubble coefficient from s-channel double cut

$$
C_{2}^{12}=-\left(y_{h}^{2} g_{1}^{2}+\frac{3 \lambda}{2}\right) C_{H B} \delta^{a \bar{a}}[12]^{2} .
$$

Following the same procedure, The contribution from t-channel cut is given by

$$
\begin{aligned}
\mathrm{Cut}^{13}\left[\mathcal{A}\left(B^{+}\left(p_{1}\right) B^{+}\left(p_{2}\right) H^{\dagger \dot{\alpha}}\left(p_{3}\right) H^{\alpha}\right)\left(p_{4}\right)\right] \\
=\mathcal{A}_{L}\left(B^{+}\left(p_{1}\right) H^{\dagger \dot{\alpha}}\left(p_{3}\right) B^{+}\left(l_{1}\right) H^{\beta}\left(l_{2}\right)\right) \delta^{\beta \dot{\beta}} \mathcal{A}_{R}\left(B^{-}\left(-l_{1}\right) H^{\dagger \dot{\beta}}\left(-l_{2}\right) B^{+}\left(p_{2}\right) H^{\alpha}\left(p_{3}\right)\right) \\
\quad+\mathcal{A}_{L}\left(B^{+}\left(p_{2}\right) H^{\alpha}\left(p_{4}\right) B^{+}\left(-l_{1}\right) H^{\dagger \dot{\beta}}\left(-l_{2}\right)\right) \delta^{\beta \dot{\beta}} \mathcal{A}_{R}\left(B^{-}\left(-l_{1}\right) H^{\beta}\left(-l_{2}\right) B^{+}\left(p_{1}\right) H^{\dagger \dot{\alpha}}\left(p_{3}\right)\right) \\
=-2 y_{h}^{2} g_{1}^{2} C_{H B} \delta^{\alpha \dot{\alpha}}\left[1 l_{1}\right]^{2} \frac{\left\langle 4-l_{1}\right\rangle\left\langle-l_{2}-l_{1}\right\rangle}{\langle 42\rangle\left\langle-\ell_{2} 2\right\rangle}+(1 \leftrightarrow 2,3 \leftrightarrow 4) .
\end{aligned}
$$

After proper parametrization and integration, we can get the bubble coefficient for $\mathrm{t}$ channel cut,

$$
C_{2}^{13}=-2 y_{h}^{2} g_{1}^{2} C_{H B} \delta^{a \bar{a}}[12]^{2} .
$$

The $\mathrm{u}$-channel cut is the same as t-channel cut under $p_{1} \leftrightarrow p_{2}$ and we get

$$
C_{2}^{13}=C_{2}^{14} \text {. }
$$

So the total contribution from bubble diagram is

$$
C_{2}=C_{2}^{13}+C_{2}^{14}+C_{2}^{12}=-\left(5 y_{h}^{2} g_{1}^{2}+\frac{3 \lambda}{2}\right) C_{H B} \delta^{\alpha \dot{\alpha}}[12]^{2} .
$$

The parts of UV divergences cancelled by IR divergences can be read from eq. (3.11) according to the external legs:

$$
C_{I R}=-(2 \gamma(B)+2 \gamma(H)) \delta^{a \dot{a}} C_{H B}[12]^{2} .
$$

So we can find the total UV divergences of operator $\mathcal{O}_{H B}$ from $\mathrm{U}(1)_{Y}$ gauge interactions at one-loop level are

$$
\begin{aligned}
C_{U V} & =C_{2}-C_{I R}=-\left[y_{h}^{2} g_{1}^{2}(5-2 \gamma(H)-2 \gamma(B))+\frac{3 \lambda}{2}\right] \frac{1}{(4 \pi)^{2}} \delta^{a \dot{a}} C_{H B}[12]^{2} \\
& =-\left[\frac{5}{3} y_{h}^{2} g_{1}^{2}+\frac{3 \lambda}{2}\right] \frac{1}{(4 \pi)^{2}} \delta^{a \dot{a}} C_{H B}[12]^{2} .
\end{aligned}
$$


So the running of $C_{H B}$ is

$$
\dot{C}_{H B}=2\left[\frac{5}{3} y_{h}^{2} g_{1}^{2}+\frac{3 \lambda}{2}\right] C_{H B}
$$

where $\dot{C}_{H B} \equiv(4 \pi)^{2} \mu \frac{d C_{H B}}{d \mu}$. This expression is exactly the same as in $[29,30]$.

\section{$4.2 \quad F^{3}$ type operators}

For the $F^{3}$ type operators, the leading amplitude is three-point scattering amplitude, which does not depend on any UV scale if the three external legs are on-shell. So its UV divergences can not be extracted through unitarity cut. However we can calculate its next leading 4-points amplitude to derive its RG running. The color-ordered leading amplitude of dimension 6 operator $\mathcal{O}_{G}=C_{G^{3}} \operatorname{Tr}\left[G_{\mu \nu}^{3}\right]$ can be expressed as

$$
\mathcal{A}\left(g_{1}^{+} g_{2}^{+} g_{3}^{+}\right)=C_{G^{3}}[12][23][31] .
$$

Its 4-point tree-level amplitudes which can be constructed through all-line shift [39],

$$
\mathcal{A}^{\text {tree }}\left(g_{1}^{+} g_{2}^{+} g_{3}^{+} g_{4}^{+}\right)=2 g_{3} C_{G^{3}} \frac{[12][13][42]}{\langle 34\rangle} .
$$

According to unitary cut, it is easy to find that the loop amplitudes of $4 g^{+}$that contain quarks internal legs does not contribute to the bubbles because the helicity selction rules forbid the tree level amplitude for two quarks and two $g^{+}$. So only $\mathcal{O}_{G}$ insertion contribute to itself RG running. Following the same procedures as above, we can get the coefficients of the massive bubble integral,

$$
C_{2}=-6 g_{3}^{2} N_{c} \mathcal{A}^{\text {tree }}\left(g_{1}^{+} g_{2}^{+} g_{3}^{+} g_{4}^{+}\right) .
$$

After including the massless bubble contributions which can be directly read from the eq. (3.11), the total UV divergences at one-loop level is

$$
C_{U V}=\frac{\left(-6 g_{3}^{2} N_{c}+4 g_{3}^{2} \gamma(g)\right)}{(4 \pi)^{2}} \mathcal{A}^{\text {tree }}\left(g_{1}^{+} g_{2}^{+} g_{3}^{+} g_{4}^{+}\right) .
$$

These divergences of this four-point amplitude contain both the gauge coupling $g_{s}$ and $C_{G^{3}}$ renormalization and with requirement that the amplitude independent on renormalizable scale we can get the following RG equation

$$
(4 \pi)^{2} \beta_{g_{3}} C_{G^{3}}+g_{3} \dot{C}_{G^{3}}=4 g_{3}^{3}\left(3 N_{c}-2 \gamma(g)\right) C_{G^{3}} .
$$

where $\beta_{g_{3}}$ is the beta function of gauge coupling $g_{3}$. We can also derive $\beta_{g_{3}}$ through on-shell method [11, 21],

$$
\beta_{g_{3}}=-\frac{2 g_{3}^{3}}{(4 \pi)^{2}} \gamma(g)
$$

Substituting the expression of $\beta_{g_{3}}$ into the eq. (4.20), we can get the RG running of $C_{G^{3}}$

$$
\dot{C}_{G^{3}}=\left[12 N_{c}-6 \gamma(g)\right] g_{3}^{2} C_{G^{3}} .
$$


With these examples we can find that on-shell method is very efficient to calculate SMEFT RG running without doing loop calculations. All the divergences can be extracted from the tree level amplitudes. We want to emphasize that although the running of the three point amplitude basis can not be derived directly via on-shell method, its running can be extracted from the running of higher point amplitude involving this basis. We also present more complicated calculation for the operator $\mathcal{O}_{e W}$ in appendix A.

\section{Anomalous dimensions at dimension 8: the $\boldsymbol{V}^{+} \boldsymbol{V}^{-} \boldsymbol{H}^{\dagger} \boldsymbol{H}$ example}

The method introduced above can be more efficient to obtain the RG running of higher dimension operators. Based on unitary cut, the some mysterious zeros of anomalous dimension can be explained by the custodial symmetry. In this section we consider the running at dimension 8. In particular we present contribution from the $H^{4}$ type local amplitude to the RG running of the coefficients of the $V^{+} V^{-} H^{\dagger} H$ type amplitude.

The amplitude $V^{+} V^{-} H^{\dagger} H$ at dimension 8 is important in phenomenology because it gives leading BSM correction to the $V V H H$ scattering in the SMEFT, due to the noninterference at dimension 6 [40]. Also this amplitude basis is only generated at one loop order when we integrate out some heavy particles in a weakly coupled UV theory [16, 4144], which makes the contribution from the mixing with a potentially tree level generated local amplitude (operator) important. So in this section we calculate the RG running of this coupling from the mixing with $H^{4}$ amplitude basis at dimension 8. Notice that if we ignore the fermions, this is the only leading contribution, all others, including the contribution from the loop containing two dimension 6 amplitude basis, are more than one loop suppressed if we take into account the tree/loop classification of the local amplitude.

There are three independent $H^{4}$ type local amplitudes at dimension 8 . It is convenient to write them in the following form:

$\mathcal{A}\left(H^{\alpha} H^{\beta} H^{\dagger \dot{\alpha}} H^{\dagger \dot{\beta}}\right)_{\operatorname{dim} 8} \supset T_{\alpha \beta \dot{\alpha} \dot{\beta}}^{+} C_{0,2}^{H^{4}+}\left(s_{13}-s_{23}\right)^{2}, T_{\alpha \beta \dot{\alpha} \dot{\beta}}^{+} C_{2,0}^{H^{4}+} s_{12}^{2}, T_{\alpha \beta \dot{\alpha} \dot{\beta}}^{-} C_{1,1}^{H^{4}-} s_{12}\left(s_{13}-s_{23}\right)$,

where $T_{\alpha \beta \dot{\alpha} \dot{\beta}}^{ \pm} \equiv \delta_{\alpha \dot{\alpha}} \delta_{\beta \dot{\beta}} \pm \delta_{\beta \dot{\alpha}} \delta_{\alpha \dot{\beta}}$.

The dimension $8 V^{+} V^{-} H^{\dagger} H$ type amplitudes can be written as:

$$
\begin{aligned}
\mathcal{A}\left(B^{+} B^{-} H^{\alpha} H^{\dagger \dot{\beta}}\right)_{\operatorname{dim} 8} & =C_{H^{2} B^{+} B^{-}} \delta_{\alpha \dot{\beta}}[1|3| 2\rangle^{2} \\
\mathcal{A}\left(W^{a+} W^{b-} H^{\alpha} H^{\dagger \dot{\beta}}\right)_{\operatorname{dim} 8} & \supset C_{H^{2} W^{+} W^{-}}^{+} T_{\alpha \dot{\beta}}^{a b+}[1|3| 2\rangle^{2}, C_{H^{2} W^{+} W^{-}}^{-} T_{\alpha \dot{\beta}}^{a b-}[1|3| 2\rangle^{2} \\
\mathcal{A}\left(W^{a+} B^{-} H^{\alpha} H^{\dagger \dot{\beta}}\right)_{\operatorname{dim} 8} & =C_{H^{2} W^{+} B^{-}} \tau_{\dot{\beta} \alpha}^{a}[1|3| 2\rangle^{2} \\
\mathcal{A}\left(B^{+} W^{a-} H^{\alpha} H^{\dagger \dot{\beta}}\right)_{\operatorname{dim} 8} & =C_{H^{2} B^{+} W^{-}} \tau_{\dot{\beta} \alpha}^{a}[1|3| 2\rangle^{2}
\end{aligned}
$$

where $\tau_{\alpha \dot{\beta}}^{a}$ is Pauli matrix, $T_{\alpha \dot{\beta}}^{a b+}=\delta^{a b} \delta_{\alpha \dot{\beta}}$ and $T_{\alpha \dot{\beta}}^{a b-}=i \epsilon^{a b c} \tau_{\alpha \dot{\beta}}^{c}$. 
The loop contributions to the running can be obtained by gluing the $H^{4}$ amplitude basis in eq. (5.1) with the $V^{+} V^{-} H^{\dagger} H$ amplitudes of SM, which are

$$
\begin{aligned}
\mathcal{A}^{S M}\left(H^{\beta}, H^{\dagger \dot{\alpha}}, B^{+}, W^{i-}\right) & =-g_{1} g_{2} y_{h}\left(\tau^{i}\right)_{\beta}^{\dot{\alpha}} \frac{\langle 14\rangle\langle 24\rangle}{\langle 13\rangle\langle 23\rangle}, \\
\mathcal{A}^{S M}\left(H^{\beta}, H^{\dagger \dot{\alpha}}, W^{+}, B^{i-}\right) & =-{ }_{1} g_{2} y_{h}\left(\tau^{i}\right)_{\beta}^{\dot{\alpha}} \frac{\langle 14\rangle\langle 24\rangle}{\langle 13\rangle\langle 23\rangle}, \\
\mathcal{A}^{S M}\left(H^{\beta}, H^{\dagger \dot{\alpha}}, W^{a+}, W^{b-}\right) & =-2 g_{2}^{2} \frac{\langle 4|1| 3]^{2}}{\langle 34\rangle[34]}\left(\frac{\left(t^{b} t^{a}\right)_{\beta}^{\dot{\alpha}}}{\langle 24\rangle[24]}+\frac{\left(t^{a} t^{b}\right)_{\beta}^{\dot{\alpha}}}{\langle 23\rangle[23]}\right),
\end{aligned}
$$

in addition to the $B^{+} B^{-} H^{\dagger} H$ amplitude in eq. (4.4). Here $t^{a}=\tau^{a} / 2$ are the $\mathrm{SU}(2)$ generators.

Applying the unitary method, we obtain the RG running of $V^{+} V^{-} H^{\dagger} H$ type operators as following:

$$
\begin{aligned}
\dot{C}_{H^{2} B^{+} B^{-}} & =-2 g_{1}^{2} y_{h}^{2}\left(C_{0,2}^{H^{4}+}+C_{2,0}^{H^{4}+}+\frac{1}{3} C_{1,1}^{H^{4}-}\right) ; \\
\dot{C}_{H^{2} B^{+} W^{-}} & =-\frac{1}{3} g_{1} g_{2} y_{h}\left(C_{0,2}^{H^{4}+}+C_{2,0}^{H^{4}+}-C_{1,1}^{H^{4}-}\right) ; \\
\dot{C}_{H^{2} W^{+} B^{-}} & =-\frac{1}{3} g_{1} g_{2} y_{h}\left(C_{0,2}^{H^{4}+}+C_{2,0}^{H^{4}+}-C_{1,1}^{H^{4}-}\right) ; \\
\dot{C}_{H^{2} W^{+} W^{-}}^{+} & =-\frac{1}{2} g_{2}^{2}\left(C_{0,2}^{H^{4}+}+C_{2,0}^{H^{4}+}+\frac{1}{3} C_{1,1}^{H^{4}-}\right) ; \\
\dot{C}_{H^{2} W^{+} W^{-}}^{-} & =0 .
\end{aligned}
$$

The dependence of the RGE on the specific combinations $\left(C_{0,2}^{H^{4}+}+C_{2,0}^{H^{4}+}+\frac{1}{3} C_{1,1}^{H^{4}-}\right)$ and $\left(C_{0,2}^{H^{4}+}+C_{2,0}^{H^{4}+}+\frac{1}{3} C_{1,1}^{H^{4}-}\right)$ are expected by demanding the right angular momentum and $\mathrm{SU}(2)$ global symmetry [16]. The zero in the last equation can be understood from the fact that the there is $\mathrm{SO}(4)$ custodial symmetry in both $H^{4}$ operators and $\mathcal{A}^{S M}\left(H^{\beta} H^{\dagger \dot{\alpha}} W^{a+} W^{b-}\right)$, while dimension 8 amplitude basis $\mathcal{A}\left(W^{a+} W^{b-} H^{\alpha} H^{\dagger \dot{\beta}}\right)_{\operatorname{dim} 8}=C_{H^{2} W^{+} W^{-}}^{-} T_{\alpha \dot{\beta}}^{a b-}[1|3| 2\rangle^{2}$ violate this symmetry. So custodial symmetry can provide some new selection rules, which can not be explained by existing selection rules, based on on-shell method.

\section{Universal results for anomalous dimension}

From above examples, we can find the form of anomalous dimension matrix is strongly dependent of the external legs of amplitude basis. With on-shell method, the structure of anomalous dimension matrix can be clearly seen and some universal results for amplitude basis RG running can thus be obtained. For example, the bubble coefficients of any four point amplitude basis are only determined by the product of two four-point on-shell scattering amplitudes. Since the four point amplitude basis with any dimension can be expressed in a uniform way: the product of spinor products and polynomial of madstam variable, $s$ and $t$, the RG running of this kind of basis should be also in the uniform form. Since in this work we focus on how to use on-shell method to derive RG running of SMEFT, we 
just give an simple example to confirm this claim. More universal results will be presented in the future work. We will show the RG running for $H^{2} B^{+} B^{-} D^{2 n}$ type amplitude basis at any dimension generated from the general $H^{4} D^{2 n+4}$ type amplitude basis. These basis can be expressed uniformly as

$$
\begin{aligned}
\mathcal{A}\left(H^{\alpha} H^{\beta} H^{\dagger \dot{\alpha}} H^{\dagger \dot{\beta}}\right) & =T_{\alpha \beta \dot{\alpha} \dot{\beta}}^{+} C_{m, 2 n}^{H^{4}+} s_{12}^{m}\left(s_{13}-s_{23}\right)^{2 n}, T_{\alpha \beta \dot{\alpha} \dot{\beta}}^{-} C_{m, 2 n+1}^{H^{4}-} s_{12}^{m}\left(s_{13}-s_{23}\right)^{2 n+1} \\
\mathcal{A}\left(B^{+} B^{-} H^{\alpha} H^{\dagger \dot{\beta}}\right) & =C_{m, n}^{H^{2} B^{+} B^{-}}\left[1\left|p_{3}\right| 2\right\rangle^{2} s_{12}^{m}\left(s_{13}-s_{23}\right)^{n} .
\end{aligned}
$$

where $T_{\alpha \beta \dot{\alpha} \dot{\beta}}^{ \pm} \equiv \delta_{\alpha \dot{\alpha}} \delta_{\beta \dot{\beta}} \pm \delta_{\beta \dot{\alpha}} \delta_{\alpha \dot{\beta}}$. Applying the on-shell method as above, we can obtain the universal RG running for $C_{m, n}^{H^{2} B^{+} B^{-}}$, compactly collected as,

$$
\begin{aligned}
\dot{C}_{m, n}^{H^{2} B^{+} B^{-}}= & -4 y_{h}^{2}\left(\sum_{i+2 j=m+n+2}^{i, j} 3 C_{i, 2 j}^{H^{4}+} F(m, n, i, 2 j)\right. \\
& \left.+\sum_{i^{\prime}+2 j^{\prime}=m+n+1}^{i^{\prime}, j^{\prime}} C_{i^{\prime}, 2 j^{\prime}+1}^{H^{4}-} F\left(m, n, i^{\prime}, 2 j^{\prime}+1\right)\right) \\
F(m, n, i, k)= & \sum_{2 g+f+h=n}^{g, f, h, l, d}\left(\frac{1}{4}\right)^{l-1}(-1)^{g} C_{i}^{2 l-d} C_{2 j}^{d} C_{l-1}^{g} C_{i-2 l+d}^{h} C_{2 l}^{l+1} C_{2 j-d}^{l} \\
& \times \int_{0}^{1}\left(1-\frac{1}{2 t}\right)^{h}\left(-\frac{1}{2 t}\right)^{m-2 l+d-h}\left(-\frac{1}{2}+\frac{t}{2}+\frac{1}{2 t}\right)^{f}\left(\frac{3}{2}+\frac{t}{3}-\frac{1}{2 t}\right)^{2 j-d-f} \\
& \times\left(\frac{1}{t}-1\right)^{l} t^{d+i} d t
\end{aligned}
$$

So the anomalous dimension for $H^{2} B^{+} B^{-}$basis at any dimension can be readily read from this universal expression. For example, the amplitude basis at dimension 8 , or $(m=0, n=$ $0)$, can get three contributions in above sum,

$$
\begin{gathered}
(i=0, j=1, d=2, l=1, g=h=f=0) \\
(i=2, j=0, d=0, l=1, g=h=f=0) \\
\left(i^{\prime}=1, j^{\prime}=0, d=1, l=1, g=h=f=0\right) .
\end{gathered}
$$

With a simple integral $\int_{0}^{1}\left(\frac{1}{t}-1\right) t^{2}=\frac{1}{6}$, we can obtain its RG running,

$$
\dot{C}_{00}^{H^{2} B^{+} B^{-}}=-2 g_{1}^{2} y_{h}^{2}\left(C_{0,2}^{H^{4}+}+C_{2,0}^{H^{4}+}+\frac{1}{3} C_{1,1}^{H^{4}-}\right),
$$

which recovers the result we obtained in previous section.

\section{Conclusion}

The on-shell amplitude methods have remarkable advantages in studying SMEFT. The nonrenormalizable interactions can be described by unfactorizable amplitude bases without worrying about redundancies in quantum field theory. Comparing with Feynman diagrams calculations, the loop-level amplitudes can be constructed by unitarity cut very efficiently, 
because there are no unphysical degrees of freedoms appearing in on-shell amplitudes. Especially, since the UV divergences of one-loop amplitudes are only from massive $\left(K_{i}^{2} \neq 0\right)$ and massless $\left(K_{i}^{2}=0\right)$ scalar bubble integrals and the bubble coefficients are related to the tree-level amplitudes, the on-shell method can be very convenient to obtain the renormalization group running of higher dimension operators (amplitude basis).

We demonstrate in detail how to extract the full UV divergences of the loop amplitudes. The UV divergences from massive bubble integrals can be extracted by unitarity cut. While the UV divergences from massless bubble integrals are canceled by collinear IR divergences and can not be obtained by unitary cut, they can be extracted from the collinear divergences of tree-level amplitudes. Since the collinear IR divergences are universal and only depend on the external legs in SMEFT, we calculate the collinear IR divergence factors for all the standard model fields and list them in eq. (3.11). So the UV divergences from the massless bubbles, which is equal to the minus of the corresponding collinear IR divergences, can be directly read from the list. Then total UV divergences, which is from both massive and massless bubble integrals, can be obtained by unitarity cuts (for massive bubble) and collinear IR divergent factors (for massless bubble).

We present some examples to show how to derive the anomalous dimension matrix correctly. The on-shell method is very powerful for SMEFT calculation. Some selection rules can be easily obtained based on this method. This method can make the structure of anomalous dimension matrix transparent, so the running of the general 4-point amplitude basis can be expressed in a universal form. We also present the universal expressions for the anomalous dimension matrix of the general amplitude basis $H^{2} B^{+} B^{-} D^{2 n}$ genrated from the contributions of general $H^{4} D^{2 n+4}$ type operators at one-loop level.

Note added. While this paper was being finalized, refs. $[45,46]$ appeared, which presents a similar topic. Ref. [45] uses both form factors and on-shell amplitudes in their calculations and gives the anomalous dimensions at two loops. Ref. [46] uses the on-shell unitary cut similar to us but considers only the mixing between different operators, for which there are no IR contributions. In this paper, we take a pure on-shell method and demonstrate the complete procedure in deriving the anomalous dimension at one loop, obtaining the bubble coefficients and subtracting the collinear divergences. We also give new results in dimension 8 as well as universal expressions in general dimensions.

\section{Acknowledgments}

We thank Rui Yu, Qingjun Jin and Gang Yang for useful discussions. J.S. is supported by the National Natural Science Foundation of China (NSFC) under grant No.11947302, No.11690022, No.11851302, No.11675243 and No.11761141011 and also supported by the Strategic Priority Research Program of the Chinese Academy of Sciences under grant No.XDB21010200 and No.XDB23000000. T.M. are supported by the United States-Israel Binational Science Foundation (BSF) (NSF-BSF program Grant No. 2018683) and the Azrieli foundation. 


\begin{tabular}{|c|c|c|}
\hline $\mathcal{A}^{6}$ & $\mathcal{A}^{\mathcal{S M}}$ & Contribution to $\dot{C}_{e W}$ \\
\hline $\mathcal{A}\left(W^{i-} W^{i-} H^{\dagger \dot{\alpha}} H^{\alpha}\right)=2\left(C_{H W}+i C_{H \tilde{W}}\right) \delta^{\alpha \dot{\alpha}}\langle 12\rangle^{2}$ & $A\left(l^{\dot{\alpha}-} e^{-} W^{i+} H^{\beta}\right)$ & $Y_{e}^{\dagger} g_{2}\left(C_{H W}+i C_{H \widetilde{W}}\right)$ \\
\hline $\mathcal{A}\left(W^{i-} B^{-} H^{\dagger \dot{\alpha}} H^{\beta}\right)\left(C_{H W B}+i C_{H \widetilde{W} B}\right)\left(\tau^{i}\right)_{\beta}^{\dot{\alpha}}\langle 12\rangle^{2}$ & $A\left(l^{\dot{\alpha}-} e^{-} B^{+} H^{\alpha}\right)$ & $Y_{e}^{\dagger}\left(y_{e}+y_{l}\right) g_{1}\left(C_{H W B}+i C_{H \widetilde{W} B}\right)$ \\
\hline $\mathcal{A}\left(l_{\dot{\alpha}}^{-} e^{-} q_{\beta}^{-} u^{-}\right)=\left[\left(C_{l e q u}^{1}-4 C_{l e q u}^{3}\right)\langle 12\rangle\langle 34\rangle-8 C_{l e q u}^{3}\langle 14\rangle\langle 32\rangle\right] \epsilon_{\dot{\alpha} \beta} \beta$ & $A^{S M}\left(u^{+} q_{\beta}^{-} W^{i-} H_{\sigma}\right)$ & $2 Y_{u} N_{c} g_{2} C_{l e q u}^{3}$ \\
\hline$A\left(l^{\dot{\alpha}-} e^{-} B^{-} H^{\alpha}\right)=-2 \sqrt{2} C_{e B} \delta^{\alpha \dot{\alpha}}\langle 13\rangle\langle 23\rangle$ & $A\left(H^{\beta} H^{\dagger \dot{\alpha}} B^{+} W^{i-}\right)$ & $2 y_{h} g_{1} g_{2} C_{e B}$ \\
\cline { 2 - 3 } & $\mathcal{A}\left(l^{+\beta} l^{-\dot{\alpha}} B^{+} W^{i-}\right)$ & $y_{l} g_{1} g_{2} C_{e B}$ \\
\hline $\mathcal{A}\left(l^{\dot{\alpha}-} e^{-} W^{i-} H^{\beta}\right)=-2 \sqrt{2} C_{e W}\left(\tau^{i}\right)_{\beta}^{\dot{\alpha}}\langle 13\rangle\langle 23\rangle$ & $\mathcal{A}\left(H^{\dagger \dot{\alpha}} H^{\alpha} e^{+} e^{-}\right)$ & $Y_{e}^{\dagger} Y_{e} C_{e W}$ \\
\cline { 2 - 3 } & $\mathcal{A}\left(l^{-\dot{\alpha}} e^{-} l^{+\alpha} e^{+}\right)$ & $4 g_{1}^{2} y_{h} y_{e}$ \\
\cline { 2 - 3 } & $\mathcal{A}\left(e^{-} e^{+} H^{\dagger \sigma} H^{\beta}\right)$ & $-4 g_{1}^{2} y_{h} y_{e}$ \\
\cline { 2 - 3 } & $A\left(l^{-\dot{\alpha}} l^{+\gamma} H^{\dagger \sigma} H^{\beta}\right)$ & $4\left(g_{2}^{2}\left(C_{2}(2)-\frac{1}{2} C_{2}(G)\right)+g^{\prime 2} y_{h} y_{l}\right)$ \\
\cline { 2 - 3 } & $\mathcal{A}\left(H^{\dagger \dot{\alpha}} W^{j+} W^{i-} H^{\beta}\right)$ & $2 g^{2}\left(C_{2}(2)+C_{2}(G)\right)$ \\
\cline { 2 - 3 } & $\mathcal{A}\left(l^{-\dot{\alpha}} l^{+\beta} W^{i-} W^{j+}\right)$ & $4 g^{2} C_{2}(2)$ \\
\hline
\end{tabular}

Table 1. Contributions to the running of $O_{e W}$ from different unitary cuts. $\mathcal{A}^{6}$ are on-shell amplitudes from dimension 6 operators and $\mathcal{A}^{S M}$ are SM amplitudes. Here $t^{j} t^{i} t^{j}=\left(C_{2}(2)-\frac{1}{2} C_{2}(G)\right) t^{i}$.

$O_{H W}=H^{\dagger} H W_{\mu \nu}^{a} W^{a \mu \nu}$
$O_{H \tilde{W}}=H^{\dagger} H \widetilde{W}_{\mu \nu}^{a} W^{a \mu \nu}$
$O_{H W B}=H^{\dagger} \tau^{a} H W_{\mu \nu}^{a} B^{\mu \nu}$
$O_{H \tilde{W} B}=H^{\dagger} \tau^{a} H \widetilde{W}_{\mu \nu}^{a} B^{\mu \nu}$
$O_{l e q u}^{1}=\left(\bar{l}^{\alpha} e\right) \epsilon_{\alpha \beta}\left(\bar{q}^{\beta} e\right)$
$O_{l e q u}^{3}=\left(\bar{l}^{\alpha} \sigma_{\mu \nu} e\right) \epsilon_{\alpha \beta}\left(\bar{q}^{\beta} \sigma^{\mu \nu} e\right)$
$O_{e B}=\left(\bar{l} \sigma^{\mu \nu} e\right) H B_{\mu \nu}$
$O_{e W}=\left(\bar{l} \sigma^{\mu \nu} e\right) \tau^{a} H W_{\mu \nu}^{a}$

\begin{tabular}{|c|c|}
\hline $\mathcal{A}\left(l^{\dot{\alpha}-} e^{-} W^{i+} H^{\beta}\right)$ & $-\sqrt{2} Y_{e}^{\dagger} g_{2} \frac{\left(\tau^{i}\right)_{\beta}^{\dot{\alpha}}}{2} \frac{\langle 12\rangle}{[12]} \frac{[23]}{\langle 13\rangle}$ \\
\hline $\mathcal{A}\left(l^{\dot{\alpha}-} e^{-} B^{+} H^{\alpha}\right)$ & $-\sqrt{2} \delta_{\alpha}^{\dot{\alpha}} Y_{e}^{\dagger} g_{1} \frac{\langle 12\rangle}{[12]}\left(y_{e} \frac{[13]}{\langle 23\rangle}+y_{l} \frac{[23]}{\langle 13\rangle}\right)$ \\
\hline $\mathcal{A}\left(u^{+} q_{\beta}^{-} W^{i-} H_{\sigma}\right)$ & $\sqrt{2} Y_{u} g_{2} \epsilon_{\lambda \sigma} \frac{\left(\tau^{i}\right)_{\beta}^{\lambda}}{2} \frac{\langle 13\rangle[21]}{[23]\langle 12\rangle}$ \\
\hline $\mathcal{A}\left(H^{\beta} H^{\dot{\dagger} \alpha} B^{+} W^{i-}\right)$ & $-g_{1} g_{2} y_{h}\left(\tau^{i}\right)_{\beta}^{\dot{\alpha}} \frac{\langle 14\rangle\langle 24\rangle}{\langle 13\rangle\langle 23\rangle}$ \\
\hline $\mathcal{A}\left(l^{+\beta} l^{\dot{\alpha}-} B^{+} W^{i-}\right)$ & $g_{1} g_{2} y_{l}\left(\tau^{i}\right)_{\beta}^{\dot{\alpha}} \frac{\langle 24\rangle^{2}}{\langle 13\rangle\langle 23\rangle}$ \\
\hline $\mathcal{A}\left(H^{\dagger \dot{\alpha}} H^{\alpha} e^{+} e^{-}\right)$ & $-Y_{e}^{\dagger} Y_{e} \frac{\langle 14\rangle}{\langle 13\rangle}$ \\
\hline $\mathcal{A}\left(l^{\dot{\alpha}-} e^{-} l^{+\alpha} e^{+}\right)$ & $2 y_{l} y_{e} g_{1}^{2} \delta_{\alpha}^{\dot{\alpha}} \frac{\langle 12\rangle[34]}{\langle 13\rangle[13]}$ \\
\hline $\mathcal{A}\left(e^{-} e^{+} H^{\dagger \dot{\sigma}} H^{\beta}\right)$ & $2 y_{h} y_{e} g_{1}^{2} \delta_{\beta}^{\dot{\sigma}} \frac{\langle 1|4| 2]}{\langle 12\rangle[12]}$ \\
\hline $\mathcal{A}\left(l^{\dot{\alpha}-} l^{+\gamma} H^{\dagger \dot{\sigma}} H^{\beta}\right)$ & $-2\left(y_{h} y_{l} g_{1}^{2} \delta_{\gamma}^{\dot{\alpha}} \delta_{\beta}^{\dot{\sigma}}+g_{2}^{2}\left(t^{i}\right)_{\gamma}^{\dot{\alpha}}\left(t^{i}\right)_{\beta}^{\dot{\sigma}}\right) \frac{\langle 1|4| 2]}{\langle 12\rangle[12]}$ \\
\hline $\mathcal{A}\left(H^{\dagger \dot{\alpha}} W^{j+} W^{i-} H^{\beta}\right)$ & $-2 g_{2}^{2} \frac{\langle 3|1| 2]^{2}}{\langle 23\rangle[23]}\left(\frac{\left(t^{i} t^{j}\right)_{\beta}^{\dot{\alpha}}}{\langle 13\rangle[13]}+\frac{\left(t^{j} t^{i}\right)_{\beta}^{\alpha}}{\langle 12\rangle[12]}\right)$ \\
\hline $\mathcal{A}\left(l^{\dot{\alpha}-} l^{+\beta} W^{i-} W^{j+}\right)$ & $2 g_{2}^{2} \frac{\langle 13\rangle[24]}{\langle 34\rangle[43]}\left(\frac{\langle 13\rangle}{\langle 14\rangle}\left(t^{j} t^{i}\right)_{\beta}^{\dot{\alpha}}+\frac{[14]}{[13]}\left(t^{i} t^{j}\right)_{\beta}^{\dot{\alpha}}\right)$ \\
\hline
\end{tabular}

Table 2. Left: dimension 6 operators that contribute to the running of $O_{e W}$; right: expressions of SM amplitudes used in calculating $\dot{C}_{e W}$.

\section{A The anomalous dimension of $\mathrm{O}_{e W}$}

In this appendix we present the full calculation of the running of $O_{e W}=\left(\bar{l} \sigma^{\mu \nu} e\right) \tau^{I} H W_{\mu \nu}^{I}$. The contribution from different unitary cut are summarized in table 1 , where $A^{6}$ and $A^{S M}$ are dimension 6 and SM on-shell amplitudes at the two sides of the cut respectively. The relevant form of dimension 6 operators and expression of SM amplitudes are summarized in table 2

Combined with the contributions from collinear divergences read from eq. (3.11):

$$
\dot{C}_{e W}=-2(\gamma(W)+\gamma(H)+\gamma(l)+\gamma(e)) C_{e W},
$$

we obtain the same result as that calculated from Feynman diagrams [28-30]. ${ }^{3}$

\footnotetext{
${ }^{3}$ There is a relative minus signs for terms linear in $g_{i}$ due to conventions.
} 
Open Access. This article is distributed under the terms of the Creative Commons Attribution License (CC-BY 4.0), which permits any use, distribution and reproduction in any medium, provided the original author(s) and source are credited.

\section{References}

[1] CMS collaboration, Observation of a new boson at a mass of $125 \mathrm{GeV}$ with the CMS experiment at the LHC, Phys. Lett. B $\mathbf{7 1 6}$ (2012) 30 [arXiv:1207.7235] [INSPIRE].

[2] ATLAS collaboration, Observation of a new particle in the search for the Standard Model Higgs boson with the ATLAS detector at the LHC, Phys. Lett. B 716 (2012) 1 [arXiv: 1207.7214] [INSPIRE].

[3] J. Wess and B. Zumino, Supergauge transformations in four-dimensions, Nucl. Phys. B 70 (1974) 39 [INSPIRE].

[4] D.V. Volkov and V.P. Akulov, Is the neutrino a Goldstone particle?, Phys. Lett. B 46 (1973) 109 [INSPIRE].

[5] D.B. Kaplan and H. Georgi, $\mathrm{SU}(2) \times \mathrm{U}(1)$ breaking by vacuum misalignment, Phys. Lett. $B$ 136 (1984) 183 [INSPIRE].

[6] H. Georgi and D.B. Kaplan, Composite Higgs and custodial SU(2), Phys. Lett. B 145 (1984) 216 [INSPIRE].

[7] M.J. Dugan, H. Georgi and D.B. Kaplan, Anatomy of a composite Higgs model, Nucl. Phys. B 254 (1985) 299 [INSPIRE].

[8] Y. Shadmi and Y. Weiss, Effective field theory amplitudes the on-shell way: scalar and vector couplings to gluons, JHEP 02 (2019) 165 [arXiv:1809.09644] [INSPIRE].

[9] T. Ma, J. Shu and M.-L. Xiao, Standard Model effective field theory from on-shell amplitudes, arXiv:1902.06752 [INSPIRE].

[10] F. Cachazo, S. He and E.Y. Yuan, Scattering of massless particles: scalars, gluons and gravitons, JHEP 07 (2014) 033 [arXiv:1309.0885] [inSPIRE].

[11] N. Arkani-Hamed, F. Cachazo and J. Kaplan, What is the simplest quantum field theory?, JHEP 09 (2010) 016 [arXiv:0808.1446] [INSPIRE].

[12] C. Cheung, K. Kampf, J. Novotny, C.-H. Shen and J. Trnka, A periodic table of effective field theories, JHEP 02 (2017) 020 [arXiv:1611.03137] [INSPIRE].

[13] I. Low, Adler's zero and effective Lagrangians for nonlinearly realized symmetry, Phys. Rev. D 91 (2015) 105017 [arXiv:1412.2145] [INSPIRE].

[14] C. Cheung and C.-H. Shen, Nonrenormalization theorems without supersymmetry, Phys. Rev. Lett. 115 (2015) 071601 [arXiv: 1505.01844] [INSPIRE].

[15] Z. Bern, J. Parra-Martinez and E. Sawyer, Nonrenormalization and operator mixing via on-shell methods, Phys. Rev. Lett. 124 (2020) 051601 [arXiv:1910.05831] [INSPIRE].

[16] M. Jiang, J. Shu, M.-L. Xiao and Y.-H. Zheng, New selection rules from angular momentum conservation, arXiv:2001.04481 [INSPIRE].

[17] Z. Bern, L.J. Dixon, D.C. Dunbar and D.A. Kosower, One loop $n$ point gauge theory amplitudes, unitarity and collinear limits, Nucl. Phys. B 425 (1994) 217 [hep-ph/9403226] [INSPIRE]. 
[18] Z. Bern, L.J. Dixon, D.C. Dunbar and D.A. Kosower, Fusing gauge theory tree amplitudes into loop amplitudes, Nucl. Phys. B 435 (1995) 59 [hep-ph/9409265] [INSPIRE].

[19] Z. Bern, L.J. Dixon and D.A. Kosower, One loop amplitudes for $e^{+} e^{-}$to four partons, Nucl. Phys. B 513 (1998) 3 [hep-ph/9708239] [InSPIRE].

[20] P. Mastrolia, Double-cut of scattering amplitudes and Stokes' theorem, Phys. Lett. B 678 (2009) 246 [arXiv:0905.2909] [INSPIRE].

[21] Y.-T. Huang, D.A. McGady and C. Peng, One-loop renormalization and the S-matrix, Phys. Rev. D 87 (2013) 085028 [arXiv:1205.5606] [INSPIRE].

[22] D. Forde, Direct extraction of one-loop integral coefficients, Phys. Rev. D 75 (2007) 125019 [arXiv:0704.1835] [INSPIRE].

[23] C. Anastasiou, R. Britto, B. Feng, Z. Kunszt and P. Mastrolia, D-dimensional unitarity cut method, Phys. Lett. B 645 (2007) 213 [hep-ph/0609191] [INSPIRE].

[24] W.T. Giele and E.W. Glover, Higher order corrections to jet cross-sections in $e^{+} e^{-}$ annihilation, Phys. Rev. D 46 (1992) 1980 [InSPIRE].

[25] W.T. Giele, E.W. Glover and D.A. Kosower, Higher order corrections to jet cross-sections in hadron colliders, Nucl. Phys. B 403 (1993) 633 [hep-ph/9302225] [INSPIRE].

[26] Z. Kunszt, A. Signer and Z. Trócsányi, Singular terms of helicity amplitudes at one loop in $Q C D$ and the soft limit of the cross-sections of multiparton processes, Nucl. Phys. B 420 (1994) 550 [hep-ph/9401294] [INSPIRE].

[27] C. Grojean, E.E. Jenkins, A.V. Manohar and M. Trott, Renormalization group scaling of Higgs operators and $\Gamma(h \rightarrow \gamma \gamma)$, JHEP 04 (2013) 016 [arXiv:1301.2588] [INSPIRE].

[28] E.E. Jenkins, A.V. Manohar and M. Trott, Renormalization group evolution of the Standard Model dimension six operators II: Yukawa dependence, JHEP 01 (2014) 035 [arXiv:1310.4838] [INSPIRE].

[29] R. Alonso, E.E. Jenkins, A.V. Manohar and M. Trott, Renormalization group evolution of the Standard Model dimension six operators III: gauge coupling dependence and phenomenology, JHEP 04 (2014) 159 [arXiv:1312.2014] [INSPIRE].

[30] E.E. Jenkins, A.V. Manohar and M. Trott, Renormalization group evolution of the Standard Model dimension six operators I: formalism and $\lambda$ dependence, JHEP 10 (2013) 087 [arXiv: 1308.2627] [INSPIRE].

[31] R. Alonso, H.-M. Chang, E.E. Jenkins, A.V. Manohar and B. Shotwell, Renormalization group evolution of dimension-six baryon number violating operators, Phys. Lett. B 734 (2014) 302 [arXiv: 1405. 0486] [InSPIRE].

[32] H.-L. Li, Z. Ren, J. Shu, M.-L. Xiao, J.-H. Yu and Y.-H. Zheng, Complete set of dimension-8 operators in the Standard Model effective field theory, arXiv:2005.00008 [INSPIRE].

[33] C.W. Murphy, Dimension-8 operators in the Standard Model effective field theory, JHEP 10 (2020) 174 [arXiv : 2005.00059] [inSPIRE].

[34] Z. Bern, L.J. Dixon and D.A. Kosower, Two-loop $g \rightarrow$ g splitting amplitudes in QCD, JHEP 08 (2004) 012 [hep-ph/0404293] [INSPIRE].

[35] Z. Bern, V. Del Duca, L.J. Dixon and D.A. Kosower, All non-maximally-helicity-violating one-loop seven-gluon amplitudes in $N=4$ super-Yang-Mills theory, Phys. Rev. D 71 (2005) 045006 [hep-th/0410224] [INSPIRE]. 
[36] R. Britto, F. Cachazo and B. Feng, Generalized unitarity and one-loop amplitudes in $N=4$ super-Yang-Mills, Nucl. Phys. B 725 (2005) 275 [hep-th/0412103] [INSPIRE].

[37] R. Britto, E. Buchbinder, F. Cachazo and B. Feng, One-loop amplitudes of gluons in SQCD, Phys. Rev. D 72 (2005) 065012 [hep-ph/0503132] [INSPIRE].

[38] H. Elvang and Y.-T. Huang, Scattering amplitudes, arXiv:1308.1697 [INSPIRE].

[39] T. Cohen, H. Elvang and M. Kiermaier, On-shell constructibility of tree amplitudes in general field theories, JHEP 04 (2011) 053 [arXiv: 1010.0257] [INSPIRE].

[40] A. Azatov, R. Contino, C.S. Machado and F. Riva, Helicity selection rules and noninterference for BSM amplitudes, Phys. Rev. D 95 (2017) 065014 [arXiv:1607.05236] [INSPIRE].

[41] N. Craig, M. Jiang, Y.-Y. Li and D. Sutherland, Loops and trees in generic EFTs, JHEP 08 (2020) 086 [arXiv : 2001.00017] [inSPIRE].

[42] C. Arzt, Reduced effective Lagrangians, Phys. Lett. B 342 (1995) 189 [hep-ph/9304230] [INSPIRE].

[43] G.F. Giudice, C. Grojean, A. Pomarol and R. Rattazzi, The strongly-interacting light Higgs, JHEP 06 (2007) 045 [hep-ph/0703164] [INSPIRE].

[44] M.B. Einhorn and J. Wudka, The bases of effective field theories, Nucl. Phys. B 876 (2013) 556 [arXiv: 1307.0478] [INSPIRE].

[45] J. Elias Miró, J. Ingoldby and M. Riembau, EFT anomalous dimensions from the S-matrix, JHEP 09 (2020) 163 [arXiv: 2005.06983] [InSPIRE].

[46] P. Baratella, C. Fernandez and A. Pomarol, Renormalization of higher-dimensional operators from on-shell amplitudes, Nucl. Phys. B 959 (2020) 115155 [arXiv:2005.07129] [InSPIRE]. 